\title{
Triplet correlations in two-dimensional colloidal model liquids
}

\author{
Carsten Ruß, Klaus Zahn and Hans-Hennig von Grünberg \\ Fachbereich Physik, Universität Konstanz, 78457 Konstanz, Germany
}

\begin{abstract}
Three-body distribution functions in classical fluids have been theoretically investigated many times, but have never been measured directly. We present experimental three-point correlation functions that are computed from particle configurations measured by means of video-microscopy in two types of quasi-two-dimensional colloidal model fluids: a system of charged colloidal particles and a system of paramagnetic colloids. In the first system the particles interact via a Yukawa potential, in the second via a potential $\Gamma / r^{3}$. Varying the particles density in the charged system, or, the interaction strength $\Gamma$ in the magnetic system, one can systematically explore how triplet correlations behave if the coupling between the particles changes. We find for both systems very similar results: on increasing the coupling between the particles one observes the gradual formation of a crystal-like local order due to triplet correlations, even though the system is still deep inside the fluid phase. These are mainly packing effects as is evident from the close resemblance between the results for the two systems having completely different pair-interaction potentials. To demonstrate that triplet correlations are significant not only locally, but also when integrated over the whole volume we consider the Born-Green equation and show that in a strongly interacting system this equation can be satisfied only with the full triplet correlation function but not with three-body distribution functions obtained from superposing pair-correlations (Kirkwood superposition approximation).
\end{abstract}

PACS numbers: 61.20.-p, 61.20.Ne, 82.70.Dd

Submitted to: J. Phys.: Condens. Matter

\section{Introduction}

The static structure of a simple fluid is commonly described in terms of the $m$-body distribution functions $g^{(m)}$, measuring the probability densities of finding two, three, and more particles at specified positions in space. The present paper is concerned with three-body distribution functions, as obtained from measured configurations in two-dimensional colloidal model fluids.

There are many examples in statistical mechanics where triplet correlations in classical fluids are important. They play an essential role, for example, in earlier theories of the pair correlation function of a fluid, such as the Born-Green-Yvon integral equation 
theory, which explicitly shows the relationsship between pair and triplet correlation functions [1, 2, 3]. Triplet correlations are furthermore used in perturbation theories for static fluid properties [4, 5, 6], in theories of transport properties [7, but also to describe solvent reorganization processes around solutes [8] or systems under shear-flow 9. 10]. And, finally, one is again led to triplet correlation functions if one is interested in the temperature or density derivative of the pair-correlation function $g^{(2)}(r) \equiv g(r)$ [11, 12].

For these and other reasons, it is not surprising to see how often triplet correlations have been theoretically investigated, in the overwhelming number of cases, by computer simulation studies. Of central importance in these studies is Kirkwood's idea [1] to approximate the triplet distribution function by three different pair distribution functions (the Kirkwood superposition approximation (KSA) explained in more detail below). The reliability and quality of the KSA has been tested extensively for pure hardsphere systems [13, 14, 15], for Lennard-Jones (LJ) fluids [16, 17, 18, 19, 20, 21, 22, 23, 24, for liquid sodium [25], but also for Yukawa fluids [26, with the general result that all qualitative features of $g^{(3)}$ are usually well described by the KSA. However, quantitative differences at small distances and high densities can be appreciable, see, for instance, 24]. Mc Neil et al. 24] compared their simulations also with analytical theories going beyond the KSA. More recent simulations aiming at an understanding of higher-order correlations are performed in 2-component asymmetric electrolyte [27, in an aqueous 1:1 electrolyte [28, and in strong 3:3 and 1:3 electrolytes [29. Finally, one has to mention theoretical work done using integral equation theory [30, 31, 17], see [23] for a review.

All these theoretical efforts contrast with the situation on the experimental side where there are only very few papers dealing with three-body correlations. One indirect way to obtain information on $g^{(3)}$ is via the isothermal pressure derivative of the fluid structure factor $\partial S(q) / \partial P$ which is related to the triplet distribution function [11]. This idea has been exploited in rare-gas fluids mainly by Egelstaff and coworkers in a series of experimental papers: in Krypton [32], Argon [33], Helium [34] and Neon [35]. But, to our knowledge, there is no direct measurement of $g^{(3)}$. It is clear where the problems come from: x-ray or neutron diffraction data provide information just on the fluid structure factor, i.e., essentially on the pair-correlation function $g(r)$. A measurement of $g^{(3)}$, however, requires the knowledge of the positions of three particles at the same time which is technically very demanding to obtain in 3D samples (see however [36]).

Here lies the advantage of video-microscopy, a modern experimental technique applied to colloidal systems to directly measure all particles' positions at all times. Essentially, one follows the phase-space trajectory of the system as it evolves in time, and has thus the same amount of information as one obtains in a simulation. It is rather straightforward to calculate higher-order correlation functions from the measured configurations. In [37, we have recently realized this idea and calculated three-body correlation functions from configurations obtained by video-microscopy from a twodimensional system of magnetic colloids. This is probably the first direct measurement of a three-body correlation function. In the present paper now we give details not 
mentioned in [37], outline a few technical points and present calculations of $g^{(3)}$ also for 2D Yukawa systems.

\section{Theoretical background}

\subsection{Distribution functions}

The radial pair and three-body distribution functions $g(r)$ and $g^{(3)}\left(\mathbf{r}_{1}, \mathbf{r}_{2}, \mathbf{r}_{3}\right)$ are used to describe the local structure of a fluid and can best be defined by means of the molecular distribution functions $n^{(m)}\left(\mathbf{r}^{m}\right)$ [12. The lowest order member of this class of functions, $n^{(1)}\left(\mathbf{r}_{1}\right) d r_{1}$, is the probability of finding a particle in the volume $d r_{1}$ at $\mathbf{r}_{1}$. In an homogeneous medium it is equal to the density $\rho . n^{(2)}\left(\mathbf{r}_{1}, \mathbf{r}_{2}\right) d r_{1} d r_{2}$ is the joint probability of finding one particle in the volume $d r_{1}$ at $\mathbf{r}_{1}$ and a second in the volume $d r_{2}$ at $\mathbf{r}_{2}$ and so on for higher orders. Dividing out the asymptotic dependency we obtain the set of $m$-body distribution functions $g^{(m)}\left(\mathbf{r}^{m}\right)$ :

$$
g^{(m)}\left(\mathbf{r}^{m}\right)=\rho^{-m} n^{(m)}\left(\mathbf{r}^{m}\right) .
$$

The first non-trivial radial distribution function is $g\left(\mathbf{r}_{1}, \mathbf{r}_{2}\right)$ which in a homogeneous, isotropic system depends only on the particle separation $r=\left|\mathbf{r}_{2}-\mathbf{r}_{1}\right|$. Next in order is $g^{(3)}\left(\mathbf{r}_{1}, \mathbf{r}_{2}, \mathbf{r}_{3}\right)$ describing the probability of finding triplets of particles. Configurations of three particles are uniquely characterized by three independent parameters (in a homogeneous, isotropic medium), which can be chosen to be the distances between the particles $r=\left|\mathbf{r}_{2}-\mathbf{r}_{1}\right|, s=\left|\mathbf{r}_{3}-\mathbf{r}_{1}\right|$ and $t=\left|\mathbf{r}_{3}-\mathbf{r}_{2}\right|$. Therefore, $g^{(3)}=g^{(3)}(r, s, t)$.

The potential of mean force is defined by

$$
\beta w^{(m)}=-\ln g^{(m)}
$$

with $\beta=1 / k T$ for the inverse thermal energy. The potentials of mean force have to be distinguished from the direct potentials; so, $w^{(2)}(r)$ should not be confused with the direct pair-potential $u(r)$ in the system. The difference between both quantities can best be understood by means of the Born-Green equation [2, 12,

$$
\frac{\partial w^{(2)}\left(r_{12}\right)}{\partial \mathbf{r}_{1}}-\frac{\partial u\left(r_{12}\right)}{\partial \mathbf{r}_{1}}=\rho \int \frac{\partial u\left(r_{13}\right)}{\partial \mathbf{r}_{1}} \frac{g^{(3)}\left(\mathbf{r}_{1}, \mathbf{r}_{2}, \mathbf{r}_{3}\right)}{g\left(r_{12}\right)} d \mathbf{r}_{3},
$$

relating the difference between the mean force and the direct pair-force to an integral over the force on particle 1 at $\mathbf{r}_{1}$ due to a particle at $\mathbf{r}_{3}$, weighted by the probability $\rho g^{(3)} d \mathbf{r}_{3} / g\left(r_{12}\right)$ of finding a particle in $d \mathbf{r}_{3}$ at $\mathbf{r}_{3}$ when it is known that other particles are located at $\mathbf{r}_{1}$ and $\mathbf{r}_{2}$. This equation is a member of the Born-Green-Yvon (BGY) hierarchy and is exact if pairwise interactions can be assumed. Inserting $\beta w^{(2)}=-\ln g$ and using the KSA as a closure relation, eq. (3) yields the BGY integral equation for $g(r)[12]$.

\subsection{Kirkwood Superposition Approximation}

In a dilute system the overall interaction is dominated by the interaction of individual

pairs. Therefore the probability of finding a certain arrangement of particles is nothing 
but the joint probability of finding individual pairs. For a triplet with particle distances $r, s$ and $t$ the approximate triplet distribution function $g_{S A}^{(3)}(r, s, t)$ is then the product of the pair distribution functions

$$
g_{S A}^{(3)}(r, s, t)=g(r) g(s) g(t) .
$$

This is the so called Kirkwood superposition approximation (KSA) 1]. Introducing the factor $G$ correcting the error made by the KSA

$$
g^{(3)}(r, s, t)=g_{S A}^{(3)}(r, s, t) G(r, s, t)
$$

and the correction potential of mean force $\Delta w^{(3)}$

$$
\Delta w^{(3)}(r, s, t)=-\ln G(r, s, t) / \beta
$$

we obtain for the triplet potential of mean force $w^{(3)}$

$$
w^{(3)}(r, s, t)=w^{(2)}(r)+w^{(2)}(s)+w^{(2)}(t)+\Delta w^{(3)}(r, s, t) .
$$

All pair correlations in $g^{(3)}$ are included in $g_{S A}^{(3)}$ while $G$ quantifies the extent of intrinsic correlations due to the simultaneous presence of a triplet of particles. For this reason, $G$ is called the triplet correlation function. In other words, if $G$ is unity everywhere, there are no genuine triplet correlations in the system, but only the trivial ones that can be expressed by pair correlation functions. $\Delta w^{(3)}$ therefore measures the extra correlation energy of three correlated particles relative to the energy of superposed correlated pairs. One should be careful not to confuse $\Delta w^{(3)}$ with a real three-body potential $u^{(3)}: \Delta w^{(3)}$ is a correlation energy and can thus have non-vanishing values even in system with particles interacting exclusively via pair-wise additive potentials $u(r)$, i.e., in cases where higher-order potentials such as $u^{(3)}$ are strictly zero.

\section{Experimental systems and technical remarks}

\subsection{Two colloidal model systems}

The triplet functions that we here present are based on a large number of particle configurations obtained from two different colloidal model systems by means of videomicroscopy. Both systems are two-dimensional (or more precisely: quasi-2D). In the first system particles interact via a tuneable magnetic dipole-dipole interaction and in the second via an electrostatic double-layer interaction.

The first system consists of paramagnetic spherical colloidal particles with an diameter of $4.7 \mu \mathrm{m}$. They are located at the bottom of an hanging water droplet whose surface can be accurately controlled to be almost perfectly flat. The positions of the particles are recorded using digital video-microscopy with subsequent image-processing on the computer. The field of view has a size of $520 \times 440 \mu \mathrm{m}$ containing typically about $10^{3}$ particles. A magnetic field $B$ applied perpendicular to the air/water interface induces in each particle a magnetic moment $M=\chi B$ which leads to a repulsive dipoledipole pair-interaction energy of

$$
\beta u(r)=\frac{\Gamma}{(\sqrt{\pi \rho} r)^{3}}
$$


with the interaction strength given by $\Gamma=\beta\left(\mu_{0} / 4 \pi\right)(\chi B)^{2}(\pi \rho)^{3 / 2}$. This is the only relevant contribution to the interparticle-potential which is hence conveniently and reversibly adjustable by varying $\Gamma$ through the external field $B$. Note in particular that by introducing $\Gamma$ we have scaled out the density. $\Gamma$ is thus the only parameter determining the phase-behavior of the system: for $\Gamma<57$ the system is liquid, for $\Gamma>60$ it is solid, and in between, i.e. for $57<\Gamma<60$, it shows an hexatic phase 38, 39. The system can be regarded as an almost ideal 2D model system as the outof-plane motion of the particles corresponds to less than $1 \%$ of their diameter. Details about the preparation of the samples and the experimental set-up can be found in [38, 39].

The second system is an aqueous suspension of highly charged sulphate-terminated PS particles of $\sigma=3 \mu \mathrm{m}$ diameter, confined between two glass plates with a $1 \mathrm{~mm}$ spacing. The particles were furthermore exposed to vertical light forces which pushed them toward the negatively-charged silica plate at the bottom of the cell, confining the system more effectively to two dimensions. The particle center positions were analyzed on-line with an imaging processing software. The particle density was varied in the system by a scanned optical laser tweezer which acts as a corral for the investigated 2D colloidal suspension. The particles interact with a screened Coulomb potential

$$
\beta u(r)=\left(\frac{Z}{1+\kappa \sigma / 2}\right)^{2} e^{\kappa \sigma} \lambda_{B} \frac{e^{-\kappa r}}{r},
$$

with the (effective) particle charge $Z$, the particle diameter $\sigma$, the Bjerrum-length $\lambda_{B}$ and the inverse Debye-screening-length $\kappa$. Via the inversion of the Ornstein-Zernicke equation for a low density system we can determine the potential quite accurately. This experiment and also the inversion procedure has been described in great detail in 40, 41. In fact, we here compute triplet correlation functions based on exactly the same set of configurations as already evaluated for other purposes in [40, 41]. Pair-correlation functions corresponding to the triplet correlation functions of this work can also be found in [40, 41].

In both systems, we made sure that the system is well equilibrated and used about 200 statistically independent configurations with approximately 500-1000 particles. We also performed standard Monte-Carlo (MC) simulations, which offers us a third way to generate the necessary set of particle configurations. The simulations produced typically 500 configurations with 2000 particles yielding much better statistics and the opportunity to check the experimental results for statistical errors.

\subsection{How to compute triplet distribution functions}

The numerical procedure for computing $g^{(3)}$ is essentially the same as the one used in simulations to determine $g(r)$. For every configuration we counted the triplets with sidelengths $r, s$ and $t$ in an array $\left[R_{i}, S_{j}, T_{k}\right]$ such that $R_{i}-\Delta R / 2 \leq r<R_{i}+\Delta R / 2$ and accordingly for $s$ and $t$ with $\Delta R=\Delta S=\Delta T$. While normalizing $g$ means each 
slot is divided by the area $A\left(R_{i}\right)=2 \pi R_{i} \Delta R$ it covers, we can write down in analogy an analytic expression for $g^{(3)}$ valid for infinitesimal small $d R$ :

$$
A_{a n}\left(R_{i}, S_{j}, T_{k}\right)=\frac{4 \pi R_{i} S_{j} T_{k} d R^{3}}{\sqrt{R_{i}^{2} S_{j}^{2}-1 / 4\left(R_{i}^{2}+S_{j}^{2}-T_{k}^{2}\right)^{2}}} .
$$

This expression also works fine for finite $\Delta R$ (replace $d R$ by $\Delta R$ in eq. (10)) except in the case of triangles with one angle close to 180 degrees. For example, a triangle with $r=1.4 \Delta R, s=1.4 \Delta R, t=2.6 \Delta R$ would be sorted into the slots $i=1, j=1, k=3$. The center values of these slots obviously do not represent a triangle and thus the normalizing procedure fails because the square root in eq. (10) turns imaginary. While we can neglect these slots for most of the plots presented, we cannot ignore these counts when integrating over the whole $g^{(3)}$ as it is done in the section on the Born-Green equation. In these cases we are not allowed to replace $d R$ by $\Delta R$ in eq. (10), but, instead, we have to compute the normalization factor numerically. For a fixed distance $r$ one can easily calculate the intersection area $A\left(r, S_{j}, T_{k}\right)$ of two circular rings with radii $S_{j}, T_{k}$ and width $\Delta R$. The numerical normalization factor $A_{n u m}\left(R_{i}, S_{j}, T_{k}\right)$ is then

$$
A_{\text {num }}\left(R_{i}, S_{j}, T_{k}\right)=2 \pi \int_{R_{i}-\Delta R / 2}^{R_{i}+\Delta R / 2} r A\left(r, S_{j}, T_{k}\right) d r .
$$

More technical details can be found in the appendix of [19].

\section{Results: Three-body distribution functions}

We first concentrate on the equilateral triangle configuration. For this special configuration we have $r=s=t$ and the superposition approximation reduces to $g_{S A}^{(3)}=g(r) g(r) g(r)=(g(r))^{3}$. Therefore, $\left(g_{S A}^{(3)}(r, r, r)\right)^{1 / 3}=g(r)$ and a comparison of $g(r)$ and $\left(g^{(3)}(r, r, r)\right)^{1 / 3}$ reveals the importance of the triplet correlation function $G(r, r, r)$ in eq. (15). Fig. (11) shows $\left(g_{S A}^{(3)}(r, r, r)\right)^{1 / 3}$ and $\left(g^{(3)}(r, r, r)\right)^{1 / 3}$ for the system with the magnetic colloids (Fig. (1/a)) and that with the charged colloids (Fig. (1/,b)). For each system type, we have analyzed three different densities ( $\Gamma$-values), where the systems are always deep in the liquid phase. These are: $\Gamma=4,14,46$ for the magnetic system and $\rho \sigma^{2}=0.037,0.167,0.186$ for the charged system.

As expected, at low density (small $\Gamma$ ) Kirkwood's superposition approximation holds very well. These are then weakly interacting systems in which particles sparsely "meet" other particles and usually interact only with a single other particle. The probability of finding a certain triplet is thus nothing but the joint probability of finding the appropriate pairs, and therefore the KSA is justified by the independence of the particular pair probabilities. Increasing the interaction strength $\Gamma$ (i.e., the density in the charged system) particles begin to interact with more than one other particle at a time. The assumption that a third particle will not interfere with the statistical distribution of the second particle begins to deteriorate. One can see that in Fig. (II): the first peak of $g^{(3)}(r, r, r)^{1 / 3}$ is always reduced in height compared to $g(r)$ and the first minimum 

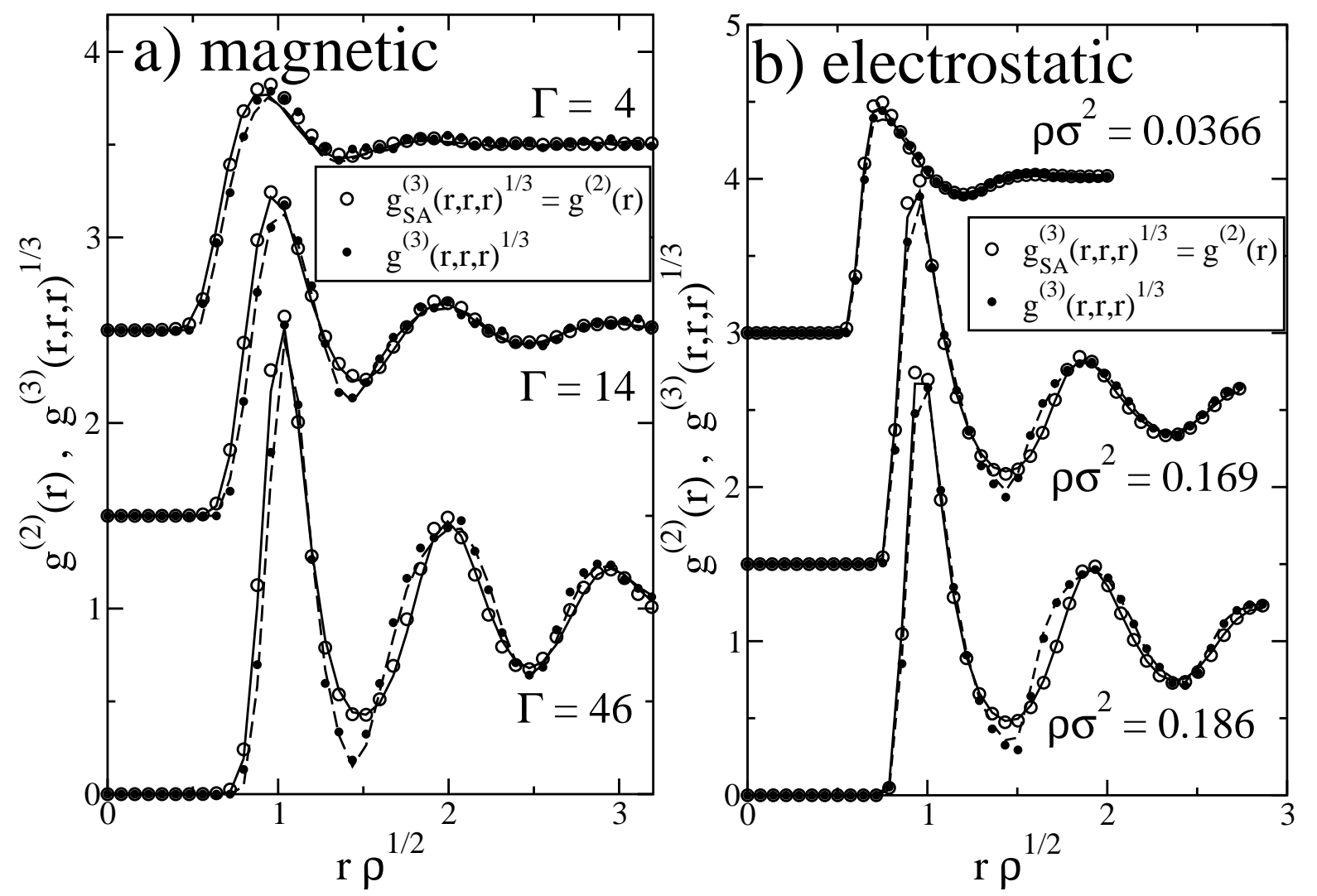

Figure 1. Comparison between triplet distribution function $g^{(3)}(r, r, r)^{1 / 3}$ and the pair-distribution function $g(r)$ of (a) a paramagnetic colloidal fluid for different $\Gamma$, and (b) the charge-stabilized colloidal fluid for different colloid densities as indicated. Circles are experimental data, lines are simulation data. The curves for different densities (different $\Gamma$ ) are shifted for clarity.

deviates even stronger. It is always more pronounced for $g^{(3)}(r, r, r)^{1 / 3}$ and it changes its shape from the sine-like oscillation of $g(r)$ to a more asymmetric one. This seem to be just a packing effect which is relatively independent of the interaction potential between the particles, as is evident from the surprisingly close resemblance between the curves in Fig. (1,a) and (1,b). Note, however, that shape and height of the first peak is different for both systems, as one would expect for different pair-potentials. The dashed and solid lines represent the results of our simulations, using the pair-potentials in eq. (8) and (9). In the charged system, the prefactor and the inverse screening length has been used as a fit-parameter. The good agreement between simulation and experimental data found for both the pair- and triplet distribution functions demonstrates that the correlations can in both systems be understood in terms of a picture of pair-wise interacting particles, although it has to be emphasized that in the charged system the pair-potential is an effective (density-dependent) one in which - through the fitting procedure - manybody contributions are absorbed into the pair-potential, see discussions in 40, 41. By contrast, many-body interactions in the magnetic system can be safely ruled out [38.

Another way to visualize $g^{(3)}(r, s, t)$ is to vary two variables while fixing one variable 


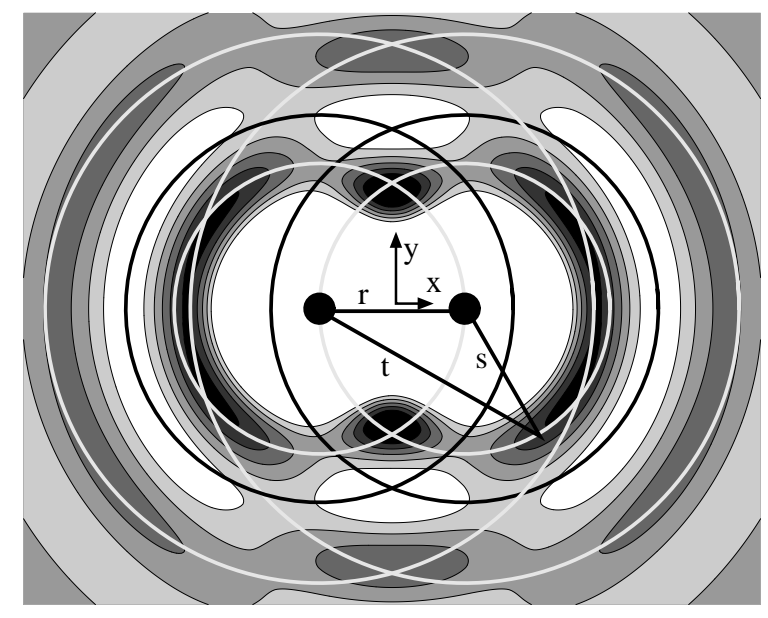

Figure 2. 2D-plot of the triplet distribution function in the Kirkwood superposition approximation, $\left(g_{S A}^{(3)}(r, s, t)\right)^{1 / 3}$, for the $\Gamma=46$-measurement (magnetic system) with $r$ fixed at the most probable two-particle separation $r_{\max }^{(2)}$. Darker areas indicate peaks in $g_{S A}^{(3)}$, circles represent the distance at which $g(r)$ around the respective particle has its first and second maximum (white circles) and its first minimum (black circles).

which we here choose to be the variable $r$. We take $r=r_{\max }^{(2)}$ which is the distance where $g(r)$ has its first peak (almost equal to $1 / \sqrt{\rho}$ ). It is the most probable distance between any pair of particles in the fluid. For this choice the KSA leads to

$$
g_{S A}^{(3)}\left(r_{\max }^{(2)}, s, t\right)=g\left(r_{\max }^{(2)}\right) g(s) g(t)
$$

and for $s \rightarrow \infty$ and $t \rightarrow \infty g_{S A}^{(3)}$ goes to the maximum value of pair correlation function, $g_{S A}^{(3)}\left(r_{\text {max }}^{(2)}, s, t\right) \rightarrow g\left(r_{\text {max }}^{(2)}\right)$. To be able to plot $g^{(3)}$ directly in the $(x, y)$-plane we have to transform the variables $s$ and $t$ accordingly, $s=s(x, y)$ and $t=t(x, y)$. Fig. (2) shows $g_{S A}^{(3)}\left(r_{\text {max }}^{(2)}, s, t\right)$ for the $\Gamma=46$ measurement of the magnetic system in the $(x, y)$ plane (co-ordinate system as defined in the plot). The lighter grey to white areas correspond to values below and the darker grey to black areas to values above the limiting value $g\left(r_{\max }^{(2)}\right)$. Clearly, the plot is highly redundant, for symmetry reasons it would have been sufficient to just show one quarter of it. In addition, we show circles with radii corresponding to the first and second maximum (white circles) and the first minimum (black circles) of $g(r)$ around each particle.

With these circles the KSA prediction can be easily understood. First we expect a very pronounced peak where the two circles with the shortest radii intersect. At this position is $s=r_{\max }^{(2)}$ and $t=r_{\max }^{(2)}$; therefore both $g(s)$ and $g(t)$ are at their maximum and that maximizes $g_{S A}^{(3)}\left(r_{\max }^{(2)}, s, t\right)$ in eq. (12). This position corresponds to an equilateral triangle configuration. Accordingly, the first minimum on the $y$-axis occurs where the two black circles intersect. Following the inner white circle around the right particle means keeping $s=r_{\max }^{(2)}$ constant. At the point where this line intersects the black circle - indicating the first minimum of $g(t)$ - we encounter a local minimum along that line. Following further the circle $s=r_{\max }^{(2)}$ the second maximum of $g(t)$ becomes important. But since the two circles intersect almost tangential there is no distinctive structure 


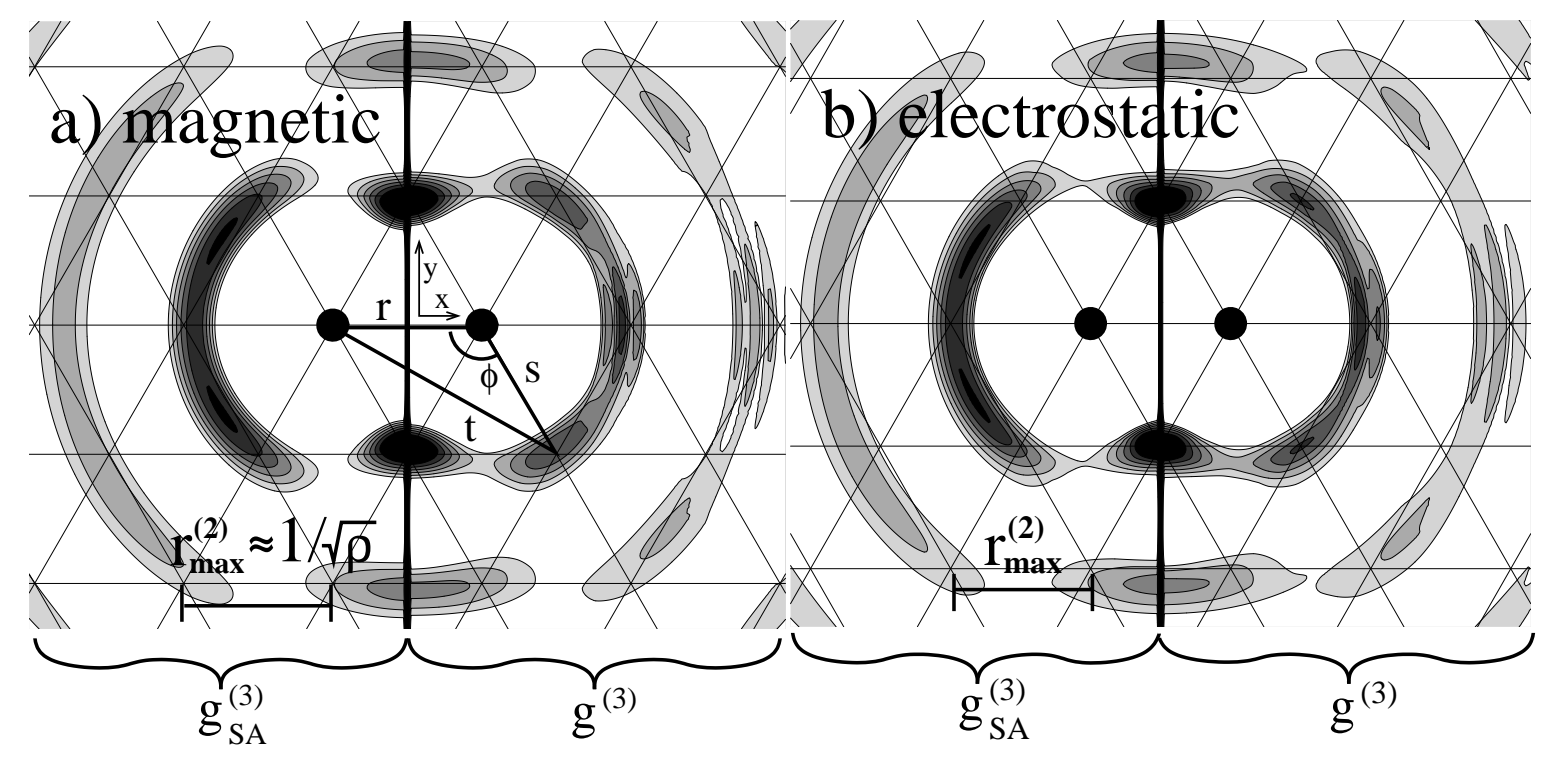

Figure 3. Comparison between the full triplet distribution function, $g^{(3)}$, in the right half of each figure and the approximated triplet distribution function $g_{S A}^{(3)}$ (Kirkwood's superposition approximation) in the left half, for (a) a paramagnetic colloidal fluid at $\Gamma=46$, and (b) the charge-stabilized colloidal fluid at a density $\rho \sigma^{2}=0.189$. The missing half of each distribution is just the mirror image of the one actually plotted. In contrast to Fig. (2), the constant $g\left(r_{\max }^{(2)}\right)$ is subtracted from the distributions and only positive values are plotted with a grey-level scheme between white (zero) and black (max. value). The underlying hexagonal lattice emphasizes approach to the crystalline phase.

but a plateau-like "banana" enclosing the particle on the outside. In a similar way, all other local maxima and minima in $g_{S A}^{(3)}$ can be explained by combining the maxima and minima of $g(t)$ and $g(s)$.

Fig. (3) compares the full triplet correlation function $g^{(3)}$ (right half of each figure) with the triplet correlation function in the KSA, $g_{S A}^{(3)}$ (left half of each figure). Again, we consider both the magnetic and the charged system, at the highest density (highest $\Gamma)$. To show the structure of $g^{(3)}$ more clearly we have set the lower end of the grey-level code to the limiting value $g\left(r_{\max }^{(2)}\right)$, so only the peaks, i.e. values where $g^{(3)}>g\left(r_{\max }^{(2)}\right)$, are shown. The stripes that can be seen especially close to the x-axis result from the transformation $g^{(3)}\left(r_{\max }, s, t\right)$ to $g^{(3)}\left(r_{\max }, x, y\right)$ and appear due to limited statistics. An hexagonal lattice with a lattice constant $a=r_{\text {max }}^{(2)}$ is superposed. As in all our plots, we plot the cubic root of $g^{(3)}$, to make the resulting numbers comparable to $g(r)$.

First of all one notices that the "banana"-like structure is not plateau-like anymore but shows a separation into three distinctive peaks. For $\phi=120^{\circ}$ (the angle $\phi$ is defined in Fig. (3) ) the peak matches almost exactly the grid point of the underlying hexagonal lattice while for $\phi=180^{\circ}$ is slightly shifted to the inside and is not so pronounced. In the shell of second nearest neighbors there is also some inner structure developing but a clear correspondence to the lattice points is not yet recognizable. By contrast, there is no such correspondence between the peak-structure of $g_{S A}^{(3)}$ and the lattice sites when the 

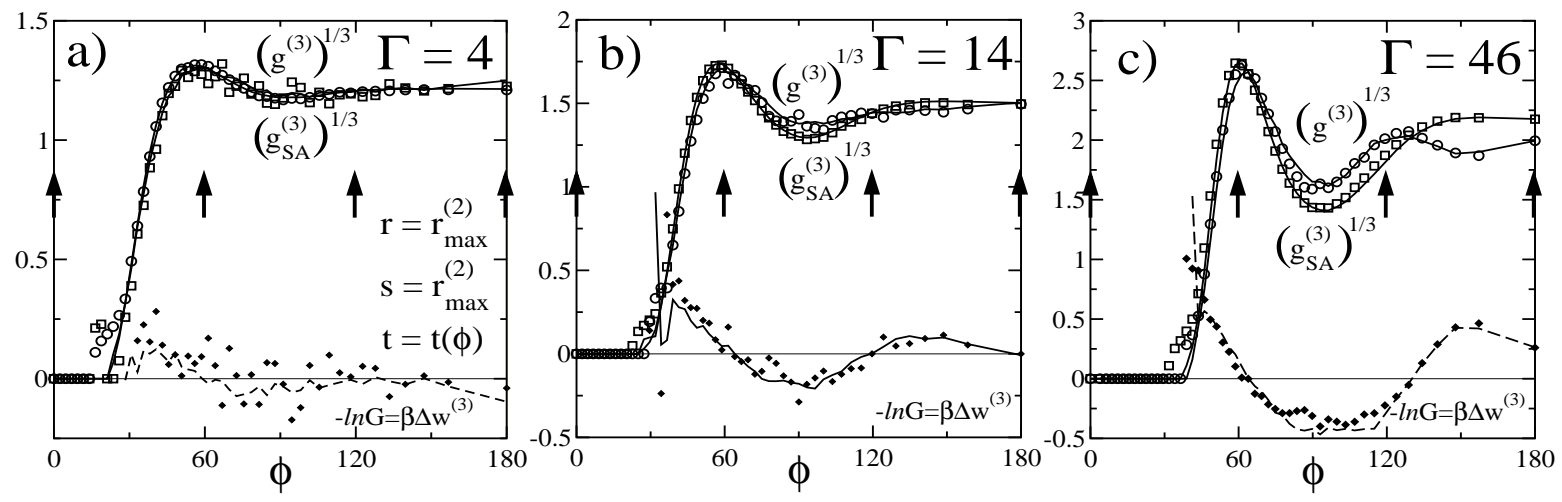

Figure 4. $g^{(3)}$ and $g_{S A}^{(3)}$, for the magnetic system, from Fig. (3) for fixed values of $r=r_{\max }^{(2)}$ and $s=r_{\max }^{(2)}$, as a function of $t=t(\phi)$ with the angle $\phi$ as defined in Fig. (3). Symbols (solid lines) for distributions generated from measured (MCsimulated) configurations. Also given is the logarithm of the triplet correlation function $G$, which is related to the triplet correlation energy $\Delta w^{(3)}$, see eq. (6). Arrows indicate positions of lattice points of the hexagonal lattice in Fig. (31)

KSA is applied. So this crystal-like local order that shows up already between particles in the fluid phase, is obviously a genuine effect of triplet correlations. Again, there is a remarkably close resemblance between the results for the magnetic and the charged system, demonstrating that these correlation effects have little to do with the properties of the pair-potential.

To quantify our analysis, we now concentrate on the first neighbor shell around the two central particles in Fig. (3) , and fix $r=r_{\max }^{(2)}$ and $s=r_{\max }^{(2)}$ while changing $t=t(\phi)$ by varying the angle $\phi$ (defined in Fig. (3)) between $0^{\circ}$ and $180^{\circ}$. Fig. (4) shows $g_{S A}^{(3)}\left(r_{\text {max }}^{(2)}, r_{\text {max }}^{(2)}, t(\phi)\right)$ and $g^{(3)}\left(r_{\text {max }}^{(2)}, r_{\text {max }}^{(2)}, t(\phi)\right)$ as a function of $\phi$, for the magnetic system. The corresponding results for the charged system look again almost identical, and need not be further considered. Varying $\phi$ and thus $t(\phi)$ while fixing $r=r_{\max }^{(2)}$ and $s=r_{\text {max }}^{(2)}$, we pass through all lattice points of the particle's first coordination shell (arrows in Fig. (4) mark positions of lattice points). It can be clearly seen that at up to $\Gamma=14 g^{(3)}$ and $g_{S A}^{(3)}$ are almost identical and that at $\Gamma=46$ the full correlation function $g^{(3)}$ shows a peak-structure in close correspondence with the hexagonal structure. This is not the case for the the approximated triplet distribution function $g_{S A}^{(3)}$.

Also given in Fig. (44) is the function $-\ln G$, i.e. $\Delta w^{(3)}$, of eq. (66). It is evident how $\Delta w^{(3)}$ gradually forms on increasing $\Gamma$, with values up to one $k T$. It is also seen that the regions of attractive and repulsive correlation energies $\Delta w^{(3)}$ correspond to the correcting effect which the function $G$ has on $g_{S A}^{(3)}$ to ensure that $g^{(3)}$ adapts locally to the hexagonal symmetry. We conclude that it is an effect entirely due to three-particle correlations, i.e. due to the function $G$, which is responsible for the observed formation of a crystal-like local environment around particles in a fluid well below the freezing transition. All quantities displayed in Fig. (44) are also compared to the corresponding results of our MC simulations, showing again a remarkably good agreement.

While Fig. (4) follows $g^{(3)}$ along a circle around the right particle in Fig. (3), Fig. (5) 


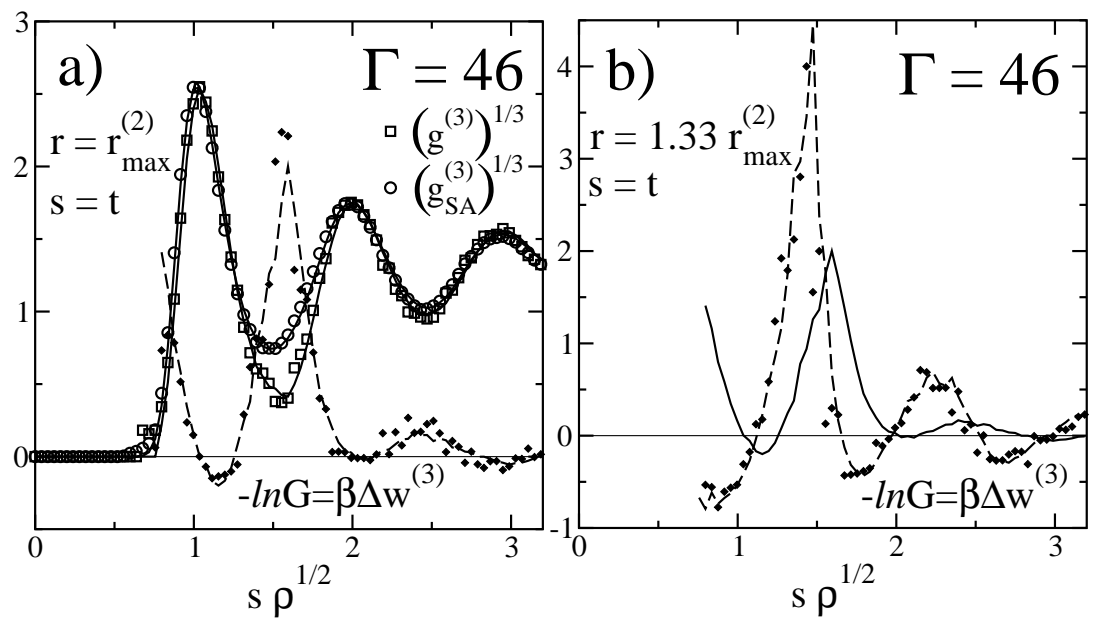

Figure 5. (a) $g^{(3)}$ and $g_{S A}^{(3)}$, for the magnetic system, as in Fig. (44), but now for $r=r_{\max }^{(2)}$ and $s=t$ (i.e. along the $y$-axis in Fig. (33)). Symbols and lines as defined in Fig. (41). (b) Solid line is the $\beta \Delta w^{(3)}$-curve of the left figure where $r=r_{\max }^{(2)}$, compared here with the corresponding quantity for the case that $r=1.33 r_{\max }^{(2)}$ (symbols: experiment, dashed line: simulation).

Figure 6. Same plot as in Fig. (3) for the magnetic system $(\Gamma=46)$, but now for different distances $r$ between the two central particles. Again, $g_{S A}^{(3)}$ in the left half of each figure, and $g^{(3)}$ in the right half.

represents a cut in the $y$-direction $(x=0)$ in Fig. (3). In this direction we have generally found the highest triplet correlation energies $\Delta w^{(3)}$. For $r=r_{\max }^{(2)} \Delta w^{(3)}$ can become as high as $2 k T$, and if $r$ is slightly increased to $1.33 r_{\text {max }}^{(2)}$, we find an even higher correlation energy of more than $4 k T$, see Fig. (5,b). Fig. (6) shows a sequence of pictures similar to that in Fig. (3). While in Fig. (3) we fixed $r$ to $r_{\max }^{(2)}, r$ is now varied. We expect triplet correlations to vanish if all three distances $r, s$ and $t$ become large, and that then $g^{(3)} \rightarrow g_{S A}^{(3)}$. Indeed, one observes from Fig. (6) that both halves of each figure (displaying $g^{(3)}$ and $g_{S A}^{(3)}$ ) show more and more resemblance the larger $s$ and $t$ are, i.e., in a region far away from the central pair, and that the extent of this region increases with increasing $r$. For example, for $r=2 r_{\max }^{(2)}$, differences between both sides are to be seen only on the midplane.

The closer the system approaches the crystalline phase the more pronounced do we expect the lattice points to be occupied. This is tested in Fig. (17) which compares the triplet distribution function for the system in the solid phase $(\Gamma=80)$ with that for the system in the liquid phase $(\Gamma=46)$. For the solid phase, we performed MC simulations, starting from a perfect hexagonal lattice, while the $\Gamma=46$ distribution is the same as in the right half of Fig. (3 a). With regard to the correlations between the central pair and the first coordination shell, there is hardly any difference between the liquid and the solid phase. Pronounced differences are observable, however, in the second shell: in the liquid phase the next nearest neighbors are broadly distributed midway between adjacent lattice nodes, while the $\Gamma=80$ distribution clearly correlates much better with 


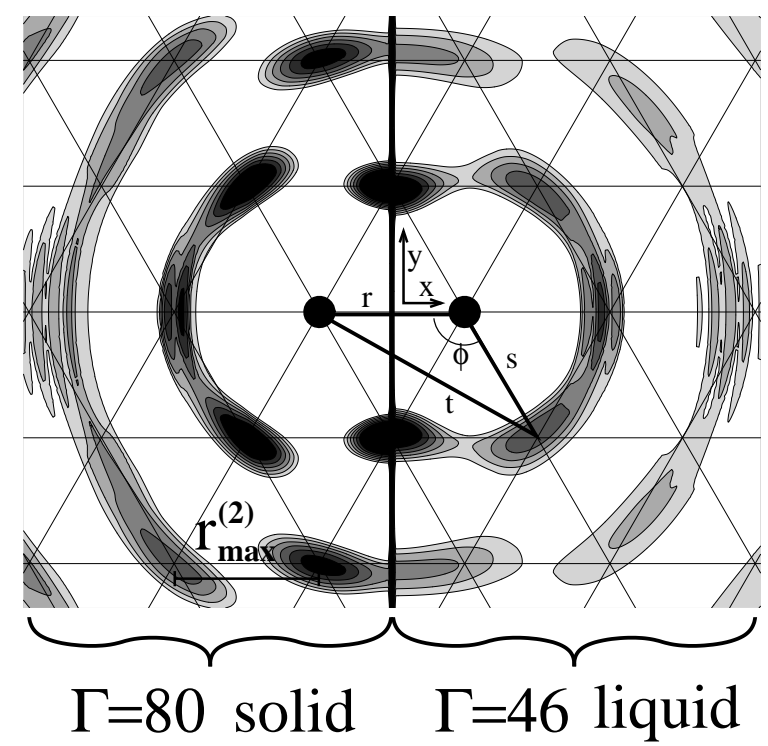

Figure 7. Distribution functions $g^{(3)}\left(r=r_{\max }^{(2)}, s(x, y), t(x, y)\right)$ for the magnetic system in the $(x, y)$-plane in the solid phase $(\Gamma=80$, left half $)$ and in the liquid phase $(\Gamma=46$, right half $)$, plotted in the same way as in Fig. (3). The $\Gamma=80(\Gamma=46)$ distribution is based on MC data (experimental data).

the lattice structure (see, for example, the lattice point in the second shell next to the mid-plane between the central pair). However, even for $\Gamma=80$ this correspondence is far from perfect; especially close to the $\phi=180^{\circ}$ direction there is still an extended smeared-out distribution showing no clear preference for certain lattice points. Clearly, approaching $T \rightarrow 0(\Gamma \rightarrow \infty)$, one will ultimately observe peaks in $g^{(3)}$ positioned exclusively on the lattice points.

Krumhansl and Wang used eq. (3) to check the accuracy of the KSA in their early simulations of a LJ fluid [20, 21]. We copy their idea, but use experimental data to demonstrate the importance of three-particle correlations. We numerically computed the right-hand side of eq. (3) using both the full and the approximated triplet function, $g^{(3)}$ and $g_{S A}^{(3)}$, of the $\Gamma=4$ and $\Gamma=46$ measurement and compare it in Fig. (8) to the left-hand side of eq. (3), evaluated using $u(r)$ and $g(r)$. For the weakly interacting system $(\Gamma=4), G$ is unity in value everywhere: triplet correlations are unimportant, and, accordingly, the Born-Green equation can be satisfied with triplet functions based on the KSA, see Fig. (8). In the strongly interacting system $(\Gamma=46)$, however, the KSA fails completely. Three-particle correlations have to be taken fully into account to obtain the correct difference between mean and direct force via the Born-Green equation.

A number of other approximations for $g^{(3)}$ are known [12, and could be checked in a similar way. This includes the Schofield equation [11], relating $\partial g(r) / \partial \rho$ to $g^{(3)}$, which in turn is the basic equation for a number of thermodynamic consistency relationships [12]. From our results, we can expect that, here again, $g^{(3)}$ at high $\Gamma$ cannot be approximated, but has to be taken in its full form. Our results confirm predictions from simulation studies on LJ and hard-sphere systems showing that qualitatively the structure of $g^{(3)}$ 


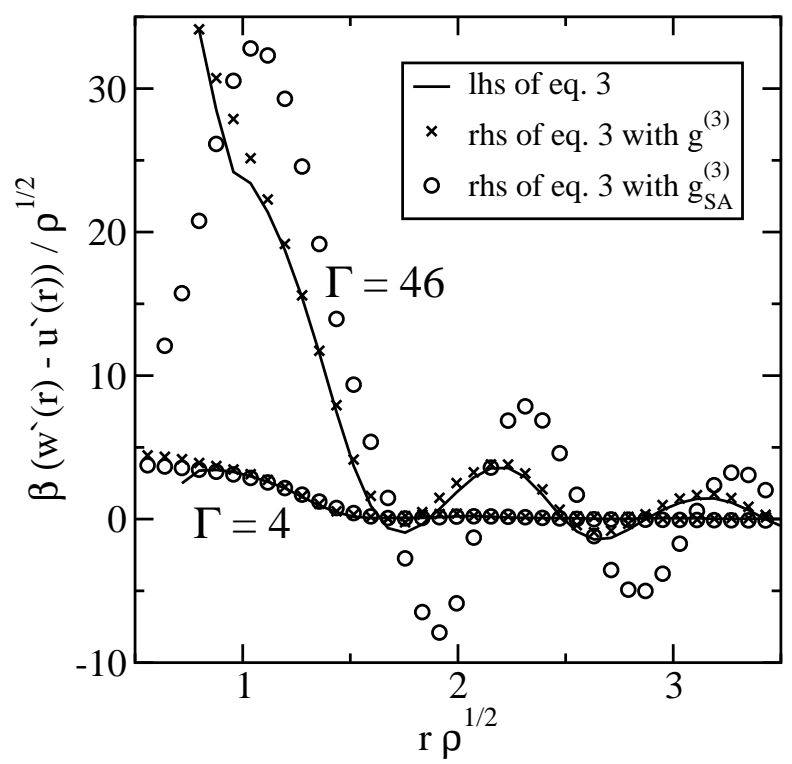

Figure 8. Test of Kirkwood's superposition approximation (KSA) by means of the Born-Green equation, eq. (3), using experimentally determined three-particle distribution functions $(\Gamma=46$ and $\Gamma=4$, magnetic system). Solid lines for the left hand side of eq. 3 symbols for the right hand side, evaluated using the full triplet distribution function $g^{(3)}$ (crosses) and the distribution function $g_{S A}^{(3)}$ in the KSA (open circles).

is correctly described by the KSA, but that quantitative failures can be appreciable [19, 24, 42, 26].

\section{Conclusion and closing remarks}

We used experimental data obtained from digital video microscopy of particles in a twodimensional colloidal model fluid. From the measured configurations, we subsequently calculated particle correlation functions; in particular, triplet correlation functions, containing much more information on the relative spatial arrangement of particles than the pair-correlation function. We studied two colloidal systems: one with particles interacting via a Yukawa pair-potential, and another with particles interacting with a $\Gamma / r^{3}$ potential. We have found very similar results for both systems: When the liquid is near the freezing transition (high density, high $\Gamma$ ), the deviations of the three-particle correlation function from unity are considerable (with correlation energies as high as $4 k T)$. We observe the formation of a crystal-like local environment around particles in a fluid well below the freezing transition. Clearly, these are packing effects as is evident from the close resemblance between the results for the two systems having completely different pair-interaction potentials. All quantities examined have also been compared to results from MC simulations, and showed always good agreement. We have finally used our experimental data in combination with the Born-Green equation to demonstrate that triplet correlations are also significant when integrated over the whole volume. For 
the strongly interacting magnetic system $(\Gamma=46)$, the KSA has been shown to fail completely. Three-particle correlations are thus seen to be important not only to obtain locally the correct structure, but also to obtain globally the correct difference between mean and direct force via the Born-Green equation.

In principle, higher-order correlation functions could be computed from the measured configurations in very much the same way, but it is not clear to us what they could be useful for. We should also remark that it soon will be possible to analyze higher-order correlation functions also in 3D colloidal samples, with positional data recorded using modern confocal microscope techniques. One has to be aware, however, that in three dimensions a similar correspondence between the peaks in $g^{(3)}$ and an underlying crystal lattice should be much harder to find. In 3D, every triplet of particle lies, of course, also in a plane, and can accordingly be plotted as in Fig. (2) and (4). However, then there is not one, but a superposition of many possible lattice planes that one has to compare this distribution with. In this respect, the role of a crystal lattice in determining the structure of a liquid is probably more pronounced in $2 \mathrm{D}$ than it is in $3 \mathrm{D}$.

\section{Acknowledgments}

HHvG and CR wish to thank Matthias Brunner and Prof. Clemens Bechinger for all the many intensive discussions on this and other topics, for their help and assistance, and the steady and close cooperation. We gratefully acknowledge continuous support and

stimulating discussion with Prof. Rudolf Klein, and financial support from the Deutsche Forschungsgesellschaft through the Konstanz SFB 513.

\section{References}

[1] J.G. Kirkwood, J. Chem. Phys. 3, 300 (1935).

[2] M. Born and H.S. Green, Proc. R. London Soc. Ser. A 188, 10 (1946).

[3] J. Yvon, Actualities Scientifiques et Industriel 203, 1 (1935).

[4] G. Stell, J.C. Rasaiah, and H. Narang, Mol. Phys. 27, 1393 (1974).

[5] W.G. Madden, D.D. Fitts, and W.R. Smith, Mol. Phys. 35, 1017 (1978).

[6] C.G. Gray, K.E. Gubbins, and C.H. Twu, J. Chem. Phys. 69, 182 (1978).

[7] K. Scherwinski, Mol. Phys. 70, 797 (1990).

[8] T. Lazaridis, J. Phys. Chem. B 104, 16 (2000).

[9] H.Wang, M.P.Lettinga, and J.K.G. Dhont, J.Phys.: Condens. Matter 14, 7599 (2002).

[10] J.K.G. Dhont and G. Nägele, Phys. Rev. E 58, 7710 (1998).

[11] P. Schofield, Proc. Phys. Soc. 88, 149 (1966).

[12] P.A. Egelstaff, An Introduction to the Liquid State (Oxford University Press, Oxford, 1992).

[13] B.J. Alder, Phys. Rev. Lett. 12, 317 (1964).

[14] S.A. Rice and J. Lekner, J. Chem. Phys. 42, 3559 (1965).

[15] E.A. Muller and K.E. Gubbins, Mol. Phys. 80, 11 (1993).

[16] A. Rahman, Phys. Rev. Lett. 12, 575 (1964).

[17] H.J. Raveche, R.D. Mountain, and W.B. Streett, J. Chem. Phys. 57, 4999 (1972).

[18] S. Gupta, J.M. Haile, and W.A. Steele, Chem. Phys. 72, 425 (1982).

[19] J.A. Krumhansl and S.S. Wang, J. Chem. Phys. 56, 2034 (1972). 
[20] J.A. Krumhansl and S.S. Wang, J. Chem. Phys. 56, 2179 (1972).

[21] S.S. Wang and J.A. Krumhansl, J. Chem. Phys. 56, 4287 (1972).

[22] R.N. Sane, Phys. Rev. A 25, 1779 (1982).

[23] H.J. Raveche and R.D. Mountain, in Progress in liquid physics, edited by C.A. Croxton (Wiley, New York, 1978).

[24] W.J. McNeil, W.G. Madden, A.D.J. Haymet, and S.A. Rice, J. Chem. Phys. 78, 388 (1983).

[25] Y.Tananka and Y. Fukui, Prog. Theor. Phys. 53, 1547 (1975).

[26] F. de J. Guevara-Rodriguez and M. Medina-Noyola, Mol. Phys. 95, 621 (1998).

[27] P. Linse, J. Chem. Phys. 94, 8227 (1991).

[28] G. Hummer and D.M. Soumpasis, J. Chem. Phys. 93, 581 (1993).

[29] S. Jorge, E. Lomba, and J.L.F. Abascal, J. Chem. Phys. 117, 3763 (2002).

[30] P. Attard, J. Chem. Phys. 95, 10 (1991).

[31] M. Fushiki, Mol. Phys. 74, 307 (1991).

[32] D.J. Winfried and P.A. Egelstaff, Can. J. Phys. 51, 1965 (1973).

[33] P.A. Egelstaff, D.I. Page, and C.R.T. Heard, Phys. Lett. A 30, 376 (1969).

[34] W. Montfrooij, L.A. de Graaf, P.J. van der Bosch, A.K. Soper, and W.S. Howells, J.Phys.: Condens. Matter 3, 4089 (1991).

[35] P.A. Egelstaff, Ann. Rev. Phys. Chem. 24, 159 (1973).

[36] S. Dietrich and W. Fenzl, Phys. Rev. B 39, 8873 (1989).

[37] K. Zahn, G. Maret, C. Russ, and H.H. von Grünberg, Phys. Rev. Lett., submitted, cond-mat 0305176, (2003).

[38] K. Zahn, R. Lenke, and G. Maret, Phys. Rev. Lett. 82, 2721 (1999).

[39] K. Zahn and G. Maret, Phys. Rev. Lett. 85, 3656 (2000).

[40] M. Brunner, C. Bechinger, W. Strepp, V. Lobaskin, and H.H. von Grünberg, Europhys. Lett. 58, 926 (2002).

[41] R. Klein, H.H. von Grünberg, C. Bechinger, M. Brunner, and V. Lobaskin, J.Phys.: Condens. Matter 14, 7631 (2002).

[42] H.J. Raveche, R.D. Mountain, and W. B. Streett, J. Chem. Phys. 61, 1970 (1974). 